Check for updates

Cite this: Mater. Chem. Front., 2019, 3, 2143

Received 19th July 2019, Accepted 19th August 2019

DOI: $10.1039 / \mathrm{c} 9 \mathrm{qm} 00471 \mathrm{~h}$

rsc.li/frontiers-materials

\title{
A glutathione responsive pyrrolopyrrolidone nanotheranostic agent for turn-on fluorescence imaging guided photothermal/photodynamic cancer therapy $\dagger$
}

\author{
Jianhua Zou, ${ }^{a}$ Lei Xue, ${ }^{a}$ Nan Yang, ${ }^{a}$ Yanfang Ren, ${ }^{\mathrm{b}}$ Zhe Fan, ${ }^{\mathrm{c}}$ Wenjun Wang, ${ }^{\mathrm{b}}$ \\ Weili Si, (D) *a Yewei Zhang, ${ }^{* c}$ Wei Huang ${ }^{\text {ade }}$ and Xiaochen Dong (ID) *a
}

\begin{abstract}
The high concentration of glutathione (GSH), a feature of solid tumors, can reduce the reactive oxygen species (ROS) damage to cancer cells, which seriously weakens the efficacy of photodynamic therapy in cancer treatment. Herein, we designed and synthesized two kinds of GSH responsive pyrrolopyrrolidone (DPP) derivatives that conjugated with 2-(diphenylmethylene) malononitrile (DPPBPh) and 2,3,3-triphenylacrylonitrile (DPPTPh), respectively. DPPTPh with two $-\mathrm{CN}$ groups shows higher singlet oxygen quantum yield and photothermal conversion efficiency than DPPBPh with four $-\mathrm{CN}$ groups. These two DPP derivatives can not only be used as colorimetric GSH probes to avoid the fluorescence quenching caused by aggregation, but also enhance the photodynamic/photothermal therapeutic efficacy of their corresponding nanoparticles (NPs). Moreover, DPPTPh NPs exhibit better therapeutic efficacy than DPPBPh NPs even at a low dose $\left(0.2 \mathrm{mg} \mathrm{kg}^{-1}\right)$. This work presents DPP derivative-based theranostic nanomedicines for GSH responsive fluorescence "turn on" imaging and enhanced photodynamic/photothermal synergistic therapy.
\end{abstract}

\section{Introduction}

Phototherapy, including photodynamic and photothermal therapy, is of great importance for cancer treatment due to its high selectivity and non-invasiveness. ${ }^{1-10}$ Different kinds of organic and inorganic materials have been utilized as photosensitizers (PSs) and achieved remarkable effectiveness in cancer diagnosis and therapy. Among these PSs, pyrrolopyrrolidone (DPP) derivatives show great potential in imaging guided phototherapy owing to their good photostability and high molar extinction coefficient. ${ }^{11-15}$ However, they usually suffer from poor solubility in aqueous solution and severe aggregation caused quenching (ACQ), which

\footnotetext{
${ }^{a}$ Key Laboratory of Flexible Electronics (KLOFE) \& Institute of Advanced Materials (IAM), Nanjing Tech University (NanjingTech), 30 South Puzhu Road, Nanjing, 211800, China.E-mail: iamxcdong@njtech.edu.cn, iamwlsi@njtech.edu.cn

${ }^{b}$ School of Physical Science and Information Technology, Liaocheng University, Liaocheng 252059, China

${ }^{c}$ Department of Hepatobiliary and Pancreatic Surgery, Zhongda Hospital, Medical School, Southeast University, Nanjing 210009, China.

E-mail: zhangyewei@njmu.edu.cn

${ }^{d}$ School of Chemistry and Materials Science, Nanjing University of Information Science \& Technology, Nanjing, 210044, China

${ }^{e}$ Shaanxi Institute of Flexible Electronics (SIFE), Northwestern Polytechnical University (NPU), 127 West Youyi Road, Xi'an, 710072, China

$\dagger$ Electronic supplementary information (ESI) available. See DOI: 10.1039/ c9qm00471h
}

limit their biological applications. Therefore, modification of pyrrolopyrrolidone (DPP) derivatives is urgently needed to develop novel PSs for overcoming aggregation caused quenching and improving anticancer efficacy.

Cancer cells with vasculature configuration and over proliferation of neoplastic cells, have an altered mitochondrial redox state, typically characterized by high levels of reactive oxygen species (ROS) and antioxidants, such as glutathione (GSH). GSH has been reported to be able to react with ROS, which can reduce the damage of ROS to the cancer cells. Therefore, it has been recognized that disrupting the redox homeostasis can be a potential strategy for cancer treatment. Depletion of antioxidants, such as GSH, will amplify intracellular oxidative stress, thereby triggering cancer cell death and enabling more effective cancer treatment.

Thiols, the typical group in GSH, have been utilized as the targeting group of diverse fluorophores, ${ }^{16-25}$ such as fluorescein, ${ }^{26}$ rhodamine ${ }^{27}$ and 4 -bora-3a,4a-diaza-s-indacene (BODIPY). ${ }^{28,29}$ The above fluorescent thiol sensors are always designed based on the photo-induced electron transfer (PET) mechanism. ${ }^{16}$ It is well known that a $-\mathrm{CN}$ group is able to recognize the thiols of GSH to form a thiazole ring via the mechanism of Michael addition, leading to an enhanced fluorescence. ${ }^{30}$ Considering the fact that the concentration of GSH in cancer cells is higher than that in normal cells, we develop two DPP derivatives, namely 
2,2' -(((5,5' -(2,5-bis(2-ethylhexyl)-3,6-dioxo-2,3,5,6-tetrahydropyrrolo[3,4-c]pyrrole-1,4-diyl)bis(thiophene-5,2-diyl))bis(3,1-phenylene))bis(phenylmethanylylidene))dimalononitrile (DPPBPh) and (2E,2'E)-3,3'-((5,5'-(2,5-bis(2-ethylhexyl)-3,6-dioxo-2,3,5,6-tetrahydropyrrolo[3,4-c]pyrrole-1,4-diyl)bis(thiophene-5,2-diyl))bis(3,1phenylene))bis(2,3-diphenylacrylonitrile) (DPPTPh) (Fig. S1, $\mathrm{ESI} \dagger$ ) for $\mathrm{GSH}$ responsive fluorescence imaging and $\mathrm{GSH}$ depletion. The nanoparticles (NPs) of the two compounds obtained by the nano-precipitation method still display high singlet oxygen generation ability and excellent photothermal conversion efficiency (34.5\% for DPPBPh and $45.2 \%$ for DPPTPh), which are desirable for photodynamic and photothermal synergistic therapy. Meanwhile, the half maximal inhibitory concentration $\left(\mathrm{IC}_{50}\right)$ of DPPTPh NPs is lower than that of DPPBPh NPs due to more -CN groups for GSH depletion. Furthermore, the fluorescence imaging indicates that both DPPTPh and DPPBPh NPs can efficiently accumulate in the tumor site via the enhanced permeability retention (EPR) effect and present GSH responsive turn-on fluorescence imaging. Moreover, DPPBPh NPs exhibit improved therapeutic efficacy compared with DPPTPh NPs even at a low dose (Scheme 1). The results provide a new way to design colorimetric photosensitizers for GSH responsive fluorescence "turn on" imaging guided phototherapy.

\section{Experimental}

\section{Materials and apparatus}

All the chemicals were purchased from Sigma (Shanghai) and used without further purification. The ${ }^{1} \mathrm{H}$ NMR and ${ }^{13} \mathrm{C}$ NMR spectra were measured on a Bruker DRX NMR spectrometer $\left(500 \mathrm{MHz}\right.$ ) at $298 \mathrm{~K}$ in $\mathrm{CDCl}_{3}$ solution with solvent residual as internal standard $\left(\mathrm{CDCl}_{3}, \delta=7.26 \mathrm{ppm}\right)$. UV-vis spectra were recorded on a UV-3600 spectrophotometer (Shimadzu, Japan). Fluorescence spectra were obtained on an F-4600 HITACHI spectrometer from Japan. Dynamic light scattering (DLS) was measured on a 90 Plus particle size analyzer from the USA

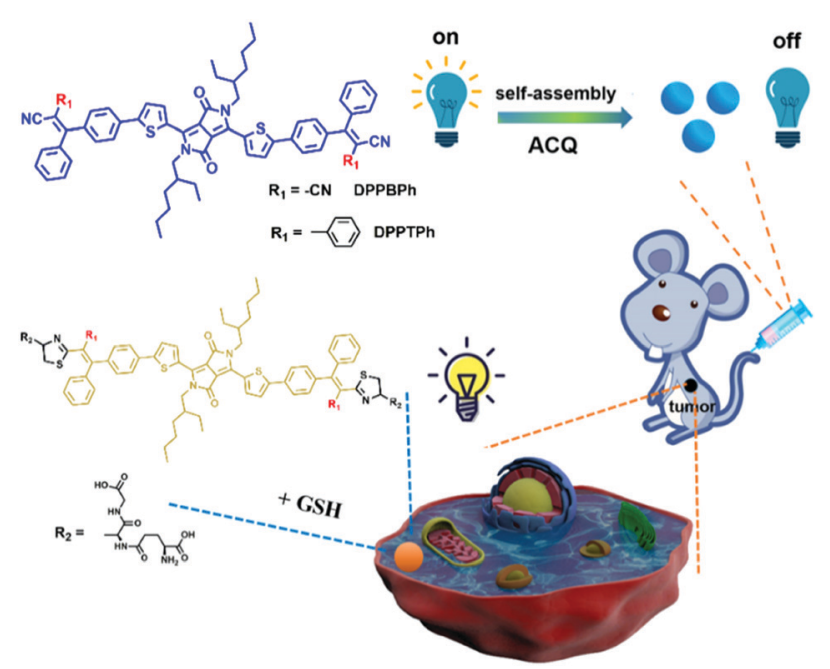

Scheme 1 Illustration of GSH responsive DPP derivatives for imaging guided phototherapy.
(Brookhaven Instruments). Transmission electron microscope (TEM) images of the NPs were measured on a JEOL JEM-2100. The fluorescence bio-imaging was recorded on a PerkinElmer IVIS Lumina K.

\section{Preparation of DPPBPh and DPPTPh NPs}

A re-precipitation method was used to prepare DPPBPh and DPPTPh NPs. DPPBPh or DPPTPh (5 mg) was dissolved in tetrahydrofuran (THF, $1 \mathrm{~mL}$ ) with ultrasonication. Then $400 \mu \mathrm{L}$ of such solution $\left(5 \mathrm{mg} \mathrm{mL}^{-1}\right)$ was added into distilled water $(10 \mathrm{~mL})$ under vigorous stirring at room temperature, respectively. Nitrogen was purged into the solution for $20 \mathrm{~min}$ to remove THF and the solution was stirred overnight. DPPBPh and DPPTPh NPs were obtained by centrifugation and re-dispersed in water.

\section{Cell culture and MTT assay}

HeLa cell lines were commercially available from Institute of Biochemistry and Cell Biology, SIBS, CAS (China). The cells were cultured in a medium consisting of Dulbecco's modified Eagle's medium (DMEM, Gibco) and fetal bovine serum (FBS, $10 \%$ ) at $37{ }^{\circ} \mathrm{C}$ under the atmosphere of $5 \% \mathrm{CO}_{2}$. The cells were seeded in a 96-well plate. Cell viability was determined by MTT (3-(4,5-dimethylthiazol-2-yl)-2,5-diphenyltetrazolium bromide) assay. Concentrated DPPBPh and DPPTPh NPs in phosphate buffer saline (PBS) were diluted with DMEM to various concentrations and added into the 96-well plate. After incubation for $24 \mathrm{~h}$, for the illumination groups, cells were irradiated with a laser $(660 \mathrm{~nm}$, $0.5 \mathrm{~W} \mathrm{~cm}^{-2}$ ) for $5 \mathrm{~min}$. While for the control or the no illumination groups, the cells were not irradiated exceptionally. After $12 \mathrm{~h}$, a solution of MTT $\left(5 \mathrm{mg} \mathrm{mL}^{-1}, 20 \mu \mathrm{L}\right)$ was added to each well under the same conditions at $37{ }^{\circ} \mathrm{C}$. Then the liquid was discarded, followed by addition of $150 \mu \mathrm{L}$ of DMSO. The absorbance (at $492 \mathrm{~nm}$ ) was measured on a microplate reader (Bio-Tek microplate) at room temperature. Cell viability was determined by the following equation: viability $(\%)=$ mean absorbance in each group with different concentrations of NPs/mean absorbance incubated with DMEM only $\times 100 \%$.

\section{Cellular uptake and in vitro ROS detection}

HeLa cells were incubated with DPPBPh and DPPTPh NPS in a confocal dish for $24 \mathrm{~h}$. Then the solution of the NPs was discarded and the cells were washed with PBS $(3 \mathrm{~mL})$, followed by treating with $1 \mathrm{~mL}$ polyoxymethylene for $25 \mathrm{~min}$. The supernatant was discarded and the cells were washed with PBS three times $(1 \mathrm{~mL})$. These samples were further incubated with 2,7-dichlorodihydrofluorescein diacetate (DCF-DA, $10 \mu \mathrm{mol}$ ) for another $3 \mathrm{~min}$ and washed with PBS three times $(1 \mathrm{~mL})$. They were irradiated by a laser $\left(660 \mathrm{~nm}, 0.5 \mathrm{~W} \mathrm{~cm}^{-2}\right)$ for 3 minutes. Fluorescence images were recorded on a confocal laser scanning microscope (CLSM). The samples incubated with DCF-DA under irradiation were excited with a $488 \mathrm{~nm}$ laser and fluorescence was collected from 500 to $600 \mathrm{~nm}$.

\section{Pharmacokinetic study}

Six Sprague-Dawley rats (three males and three females, $220 \pm 10.0 \mathrm{~g}$ ) were obtained from the Animal Center of Nanjing 
Medical University (NJMU, Nanjing, China). The animals were housed under standard conditions and had free access to food and water. This animal study was reviewed and approved by the Animal Ethical Committee of Nanjing Tech University. After being fasted for $12 \mathrm{~h}$, six rats were injected with DPPBPh, DPPTPh NPs, and molecular solutions dissolved in saline containing $10 \%$ DMSO, respectively, at the dose of $10 \mathrm{mg} \mathrm{kg}^{-1}$ via the tail vein. Blood samples $(0.2 \mathrm{~mL})$ were collected in heparinized Eppendorf tubes from the ophthalmic venous plexus at 0 (pre-dose), $0.083,0.25,0.5,1,2,4,6,8,12$ and $24 \mathrm{~h}$ after intravenous (i.v.) administration. Plasma was separated by centrifuging the blood at $3000 \times g$ for $10 \mathrm{~min}$ at $4{ }^{\circ} \mathrm{C}$ and stored at $-70{ }^{\circ} \mathrm{C}$ until analysis.

The dispersions of DPPBPh and DPPTPh NPs at different concentrations were prepared by diluting stock solution with DMSO, while the working solutions for DPPBPh and DPPTPh NPs were diluted by PBS at the same range. Subsequently, the calibration standards were prepared by spiking $20 \mu \mathrm{L}$ DPPBPh and DPPTPh NPs into $180 \mu \mathrm{L}$ blank rat plasma at concentrations of $1.25,2.5,5,10,20$, and $50 \mu \mathrm{g} \mathrm{mL} \mathrm{m}^{-1}$. The standard curve samples and the rat plasma samples were transferred to the micro cuvette and analyzed by using an ultraviolet spectrophotometer.

The pharmacokinetic parameters were calculated by a noncompartmental method using DAS 3.0 software (Mathematical Pharmacology Professional Committee of China, Shanghai, China). The area under the curve (AUC) from zero to the last measurable plasma concentration point $\left(\mathrm{AUC}_{0-t}\right)$, AUC from zero to infinity $\left(\mathrm{AUC}_{0-\infty}\right)$, the elimination rate constant $(K)$, the terminal elimination half-life $\left(T_{1 / 2}\right)$, mean residence time (MRT), and systemic clearance (CL) were calculated by software.

\section{In vivo tumor treatment histology examination and fluorescence imaging}

The animal ethic approval was available from the Animal Center of Nanjing Technological University (Nanjing, China, SCXK-2017-007). 20 nude mice were injected with HeLa cells into the armpit as the tumor source. The mice were divided into 5 groups randomly when the tumor volume reached approximately $100 \mathrm{~mm}^{3}$. The mice in the control group were intravenously injected with saline and the illumination groups were intravenously injected with DPPBPh and DPPTPh NPs $\left(50 \mu \mathrm{g} \mathrm{mL}^{-1}, 100 \mu \mathrm{L}\right)$, respectively. The tumors of the control and illumination groups were irradiated by laser $(660 \mathrm{~nm}$, $0.5 \mathrm{~W} \mathrm{~cm}^{-2}$ ). Every two days, the body weight and tumor volume of these mice were recorded. The tumor volume was calculated with the following equation: volume $=$ length $\times$ width $^{2} \times 0.5$. The nude mice were then sacrificed after phototherapy. The tumors and normal organs, including heart, liver, spleen, lungs and kidneys, were analyzed by histology. The tissues of the tumor and main organs were embedded in paraffin cassettes and stained with hematoxylin and eosin (H\&E) after dehydration, and the images were recorded on a microscope.

\section{Statistical analysis}

Data were presented as mean \pm standard error of the mean (SEM). Significant differences between groups were indicated by ${ }^{*} p<0.05,{ }^{* *} p<0.01$ and ${ }^{* *} p<0.001$, respectively.

\section{Results and discussion}

\section{Synthesis and characterization of DPPBPh and DPPTPh}

The general synthetic routes of DPPBPh and DPPTPh are shown in the ESI, $\dagger$ (Fig. S1). DPPBPh and DPPTPh were prepared through a $\mathrm{C}-\mathrm{H}$ activation reaction in the presence of anhydrous $\mathrm{K}_{2} \mathrm{CO}_{3}$ and $\mathrm{PivOH}$ with $\mathrm{Pd}(\mathrm{OAc})_{2}$ as the catalyst in anhydrous DMA solution. These two compounds could be obtained in moderate yields. The structures of DPPBPh and DPPTPh have been characterized by ${ }^{1} \mathrm{H}$ NMR, ${ }^{13} \mathrm{C}$ NMR and mass spectroscopy (Fig. S2-S5, ESI $\dagger$ ). As shown in Fig. 1a, DPPBPh and DPPTPh in THF exhibit maximum absorbance at $575 \mathrm{~nm}$, $612 \mathrm{~nm}$ and $573 \mathrm{~nm}, 612 \mathrm{~nm}$, respectively. The absorbance peaks of the as-prepared nanoparticles shift to $614,672 \mathrm{~nm}$ for $\mathrm{DPPBPh}$ and 594, $655 \mathrm{~nm}$ for DPPTPh in water, respectively. In the photoluminescence spectra (Fig. 1b), DPPBPh and DPPTPh in THF show emission peaks at $649 \mathrm{~nm}, 710 \mathrm{~nm}, 651 \mathrm{~nm}$, and $711 \mathrm{~nm}$, respectively. In contrast, a large Stokes shift was also observed for those nanoparticles in water $(727 \mathrm{~nm}, 808 \mathrm{~nm}$ for DPPBPh and $728 \mathrm{~nm}, 808 \mathrm{~nm}$ for DPPTPh), which can be ascribed to either the solvent effect or the aggregation of these compounds. The photograph of the compounds in THF and the NPs in water is shown in Fig. 1c.

High production of ${ }^{1} \mathrm{O}_{2}$ is of tremendous significance for photosensitizers. The ${ }^{1} \mathrm{O}_{2}$ QYs of DPPBPh and DPPTPh in dichloromethane (DCM) were quantitatively calculated using methylene blue (MB) as a standard substance $\left(\Phi_{\Delta}=57 \%,{ }^{3}\right.$ Fig. S6, ESI $\left.\dagger\right)$.
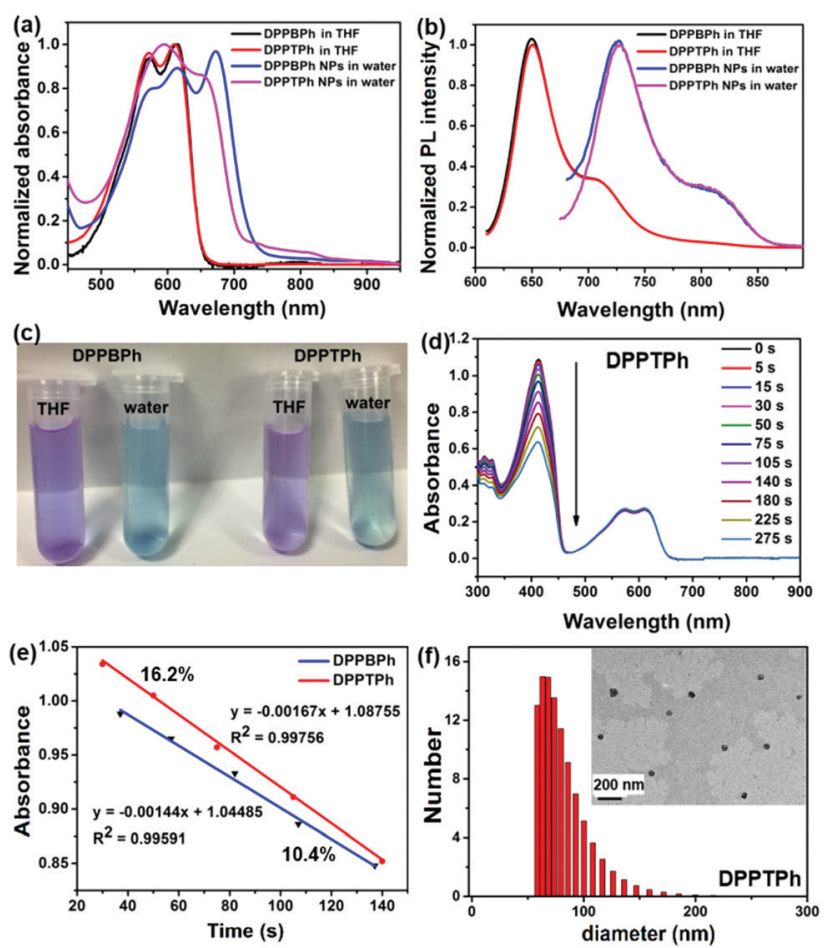

Fig. 1 ( $a$ and b) Normalized absorbance and emission spectra of DPPBPh and DPPTPh in THF and NPs in water. (c) Photograph of DPPBPh, DPPTPh in THF and their corresponding NPs in water. (d) Degradation of DPBF in the presence of DPPTPh under irradiation. (e) Linear fitting of degradation of absorbance. (f) DLS and TEM of DPPTPh NPs in water. 
The degradation speed of 1,3-diphenylisobenzofuran (DPBF) with DPPTPh is higher than that of DPPBPh. And the ${ }^{1} \mathrm{O}_{2}$ QY of DPPTPh was determined to be $22.3 \%$, which is superior to that of DPPBPh (12.5\%) (Fig. 1d and e). The morphology and size of DPPBPh and DPPTPh NPs were studied by transmission electron microscope (TEM) and dynamic light scattering (DLS), respectively. From Fig. 1f and Fig. S6d (ESI $\dagger$ ), it can be seen that these compounds formed spherical NPs with hydrodynamic size distribution between 34 to $135 \mathrm{~nm}$ for DPPBPh and 58 to $192 \mathrm{~nm}$ for DPPTPh, respectively.

\section{Photothermal conversion efficiency of DPPBPh and DPPTPh NPs}

To examine the photothermal conversion efficiency, the temperature changes of DPPBPh and DPPTPh NPs in water were recorded under laser irradiation $\left(660 \mathrm{~nm}, 0.5 \mathrm{~W} \mathrm{~cm}^{-2}\right.$ ). As illustrated in Fig. 2a, the temperature increase of DPPBPh and DPPTPh NP dispersions are $34.8{ }^{\circ} \mathrm{C}$ and $17.2{ }^{\circ} \mathrm{C}$, respectively, while that of water is almost negligible. The photothermal conversion efficiency of DPPTPh NPs was calculated to be $45.2 \%$, which was higher than that of DPPBPh NPs (34.5\%) (Fig. $2 b$ and c). The singlet oxygen quantum yield of DPPBPh and DPPTPh is lower than that of the previously reported 3,6-bis(5-(4-(diphenylamino)-phenyl)thiophen-2-yl)-2,5-bis(2-ethyl heptyl)pyrrolo[3,4-c]pyrrole-1,4 $(2 H, 5 H)$-dione (DPPTPA) NPs (33.6\%). And the photothermal conversion efficiency of DPPBPh is parallel to
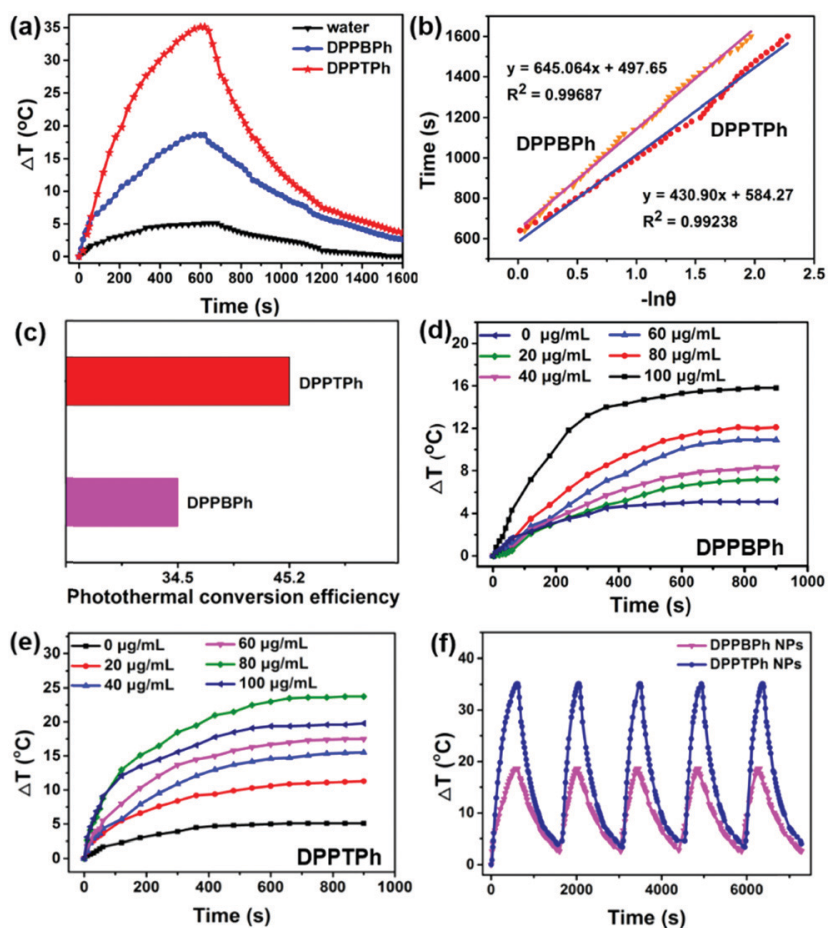

Fig. 2 Photothermal effect of the DPPBPh and DPPTPh NPs. (a) Temperature increase of water, DPPBPh and DPPTPh NPs under $660 \mathrm{~nm}$ laser irradiation $\left(0.5 \mathrm{~W} \mathrm{~cm}^{-2}, 12 \mathrm{~min}\right)$ and cooling to room temperature. (b) Linear fitting of $-\ln \theta$ and time in the cooling curve of DPPBPh and DPPTPh NPs. (c) Photothermal conversion efficiency of DPPBPh and DPPTPh NPs. ( $d$ and e) Temperature increase of DPPBPh and DPPTPh NPs at different concentrations under laser irradiation $\left(660 \mathrm{~nm}, 0.5 \mathrm{~W} \mathrm{~cm}^{-2}\right)$. (f) Cyclic temperature variation of DPPBPh and DPPTPh NPs after irradiation and cooling 5 times. that of DPPTPA (34.5\%) while that of DPPTPh is higher. $^{15}$ In addition, the temperature increased with the increasing of the DPPBPh and DPPTPh NP concentrations, indicating obvious concentration dependence (Fig. 2d and e). Meanwhile, both the DPPBPh and DPPTPh NPs exhibited outstanding optical stability even after 5 cycles of laser-induced photothermal heating (Fig. 2f), demonstrating excellent photothermal stability.

\section{GSH responsiveness of DPPBPh and DPPTPh}

Due to the strong $\pi-\pi$ stacking interaction between DPP molecules, they tend to suffer from severe fluorescence quenching in the aggregated state (aggregation caused quenching), which is disadvantageous for fluorescence imaging. Owing to the high GSH concentration in the tumor microenvironment (TME), the $-\mathrm{CN}$ of DPPBPh and DPPTPh can be used as the recognition site towards GSH, and lead to the fluorescence "on" state upon reaction with intracellular GSH. To investigate the GSH response property of these two compounds, the absorbance and fluorescence changes were measured before and after addition of GSH. As illustrated in Fig. 3a and b, the color of DPPBPh and DPPTPh changed from blue to yellow after reaction with GSH for $4 \mathrm{~h}$, in accordance with the blue shift of the absorbance. Surprisingly, the fluorescence intensity of DPPBPh and DPPTPh after reaction with GSH was 6 and 8 times higher than that of the original ones, indicating that the two compounds can be used as colorimetric GSH probes to avoid the fluorescence quenching caused by aggregation (Fig. $3 \mathrm{c}$ and $\mathrm{d}$ ). The mechanism can be ascribed to the Michael addition reaction of $-\mathrm{SH}$ and $-\mathrm{CN}$ groups to form a thiazole.

\section{In vitro experiment}

High phototoxicity as well as low dark toxicity is essential for an ideal photosensitizer. As demonstrated by MTT assay, both the DPPBPh NPs and DPPTPh NPs showed negligible cytotoxicity at low concentrations. However, with the increase of concentration,
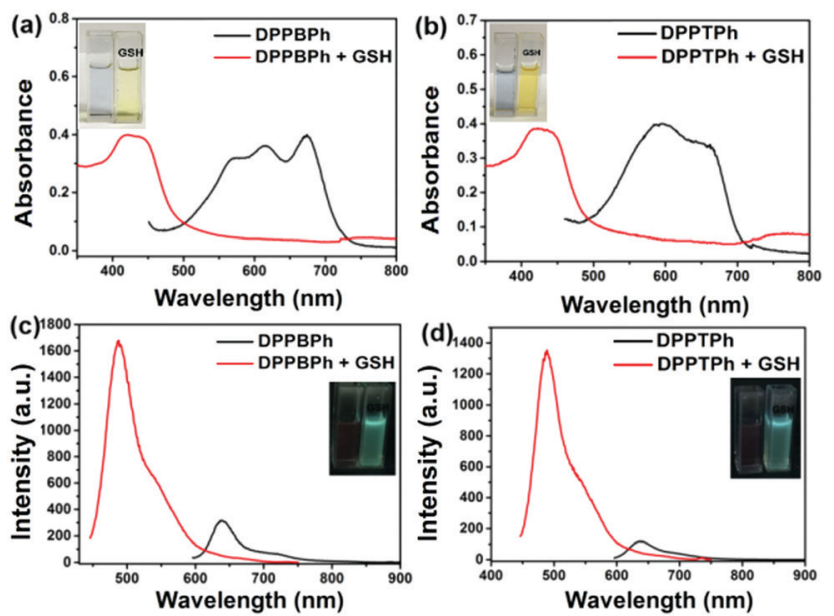

Fig. 3 (a and b) Absorbance spectra of DPPBPh $\left(1 \times 10^{-5} \mathrm{M}\right)$ and DPPTPh $\left(1 \times 10^{-5} \mathrm{M}\right)$ with and without GSH $\left(2 \times 10^{-5} \mathrm{M}\right)$. (c and d) Fluorescence spectra of DPPBPh $\left(1 \times 10^{-5} \mathrm{M}\right)$ and DPPTPh $\left(1 \times 10^{-5} \mathrm{M}\right)$ after reaction with GSH $\left(2 \times 10^{-5} \mathrm{M}\right)$ for $4 \mathrm{~h}$ in a mixture of $\mathrm{CH}_{3} \mathrm{CN} /$ water $(1: 3)$; the black lines correspond to the control groups without GSH. 

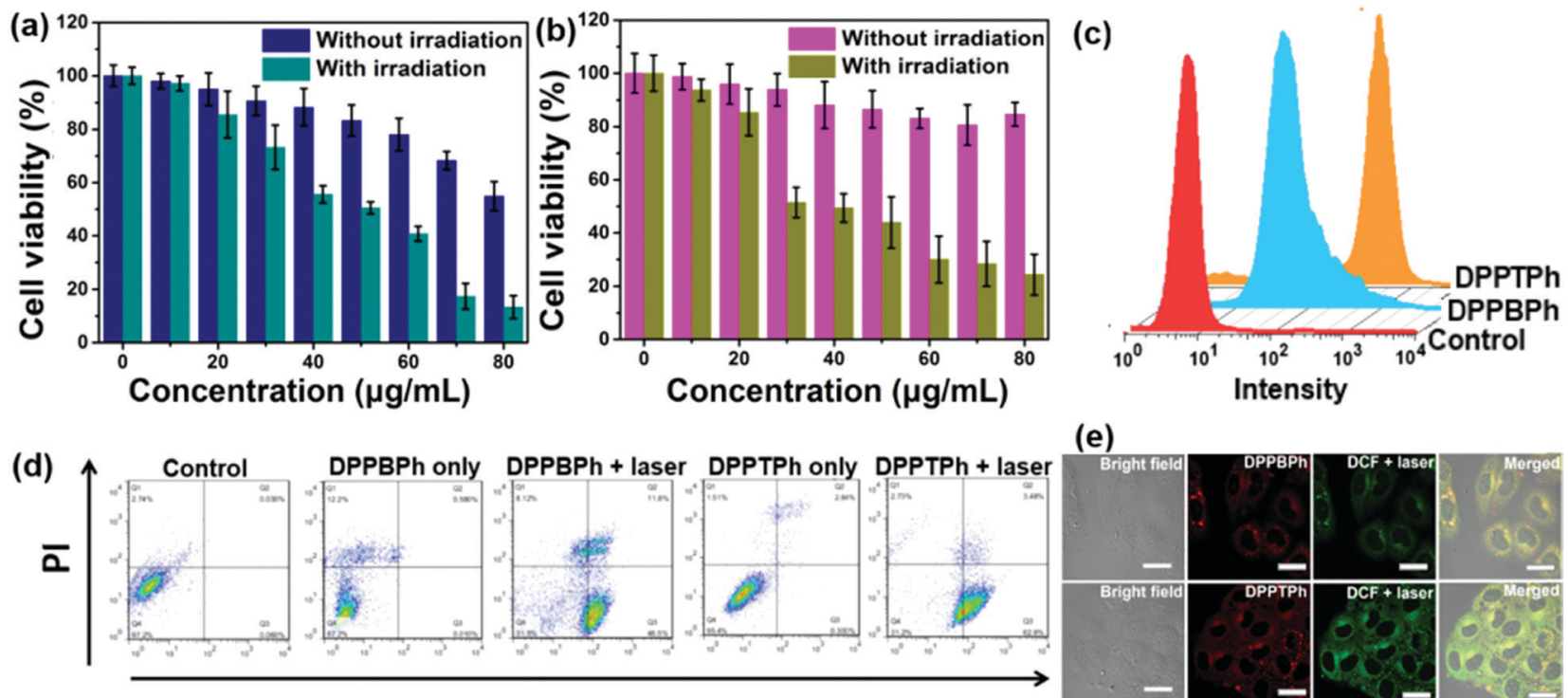

(e)

\section{ANNEXIN-V-FITC}

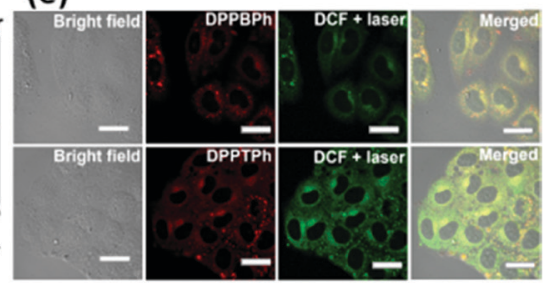

Fig. 4 MTT assay of HeLa cells after incubation with (a) DPPBPh NPs or (b) DPPTPh NPs for $24 \mathrm{~h}$ with or without laser irradiation (660 nm, $8 \mathrm{~min}, 0.5 \mathrm{~W} \mathrm{~cm}^{-2}$ ). (c) Fluorescence intensity of the control, DPPBPh NP and DPPTPh groups in HeLa cells with flow cytometry. (d) Annexin-V/PI double staining assay for HeLa cells treated with DPPBPh NPs and DPPTPh NPs with or without irradiation by flow cytometry. (e) Confocal images of DCF stained HeLa cells treated with DPPBPh NPs and DPPTPh NPs under laser irradiation $\left(660 \mathrm{~nm}, 8 \mathrm{~min}, 0.5 \mathrm{~W} \mathrm{~cm}^{-2}\right)$.

the DPPBPh NPs exhibited relatively higher dark toxicity to cancer cells compared with DPPTPh NPs, which might be due to more -CN groups in DPPBPh NPs than in DPPTPh NPs (Fig. 4a and b). DPPTPh NPs could kill cancer cells with higher efficiency than DPPBPh NPs under laser irradiation, resulting from higher ${ }^{1} \mathrm{O}_{2}$ QY and photothermal conversion efficiency of DPPTPh NPs. Flow cytometry analysis confirmed that the NPs can be uptaken by HeLa cells and the fluorescence intensity of the groups incubated with DPPBPh NPs or DPPTPh NPs is much higher than that of the control group (Fig. 4c). Apoptosis assay after nanoparticle treatment and irradiation (Fig. 4d) confirmed that the apoptosis rates of DPPBPh NPs and DPPTPh NPs were 52.3\% and 65.7\%, respectively, which is consistent with the MTT assay. A confocal laser scanning microscope (CLSM) was used to investigate the cellular uptake and ROS generation in vitro. Fig. 4e shows that these NPs can be effectively uptaken by HeLa cells by observing the red fluorescence upon excitation at $633 \mathrm{~nm}$. When 2,7-dichlorofluorescein diacetate (DCFH-DA) was added as an ROS probe, strong green fluorescence upon excitation at $488 \mathrm{~nm}$ could be observed in the cancer cells incubated with DPPBPh NPs or DPPTPh NPs after laser irradiation, suggesting the excellent ROS generation ability of DPPBPh NPs and DPPTPh NPs.

\section{In vivo fluorescence imaging and photothermal imaging}

To investigate the tumor accumulation of DPPBPh and DPPTPh NPs in vivo, fluorescence imaging was utilized. HeLa cell xenograft mice were intravenously injected with DPPBPh and DPPTPh NPs $\left(100 \mu \mathrm{g} \mathrm{mL} \mathrm{mL}^{-1}, 100 \mu \mathrm{L}\right)$, respectively. Distribution of the NP fluorescence was imaged both in vivo and ex vivo. As illustrated in Fig. 5a, the fluorescence intensity gradually increased and reached the maximum at $8 \mathrm{~h}$ for DPPBPh NPs and $4 \mathrm{~h}$ for DPPTPh NPs, respectively. After $24 \mathrm{~h}$ post injection,

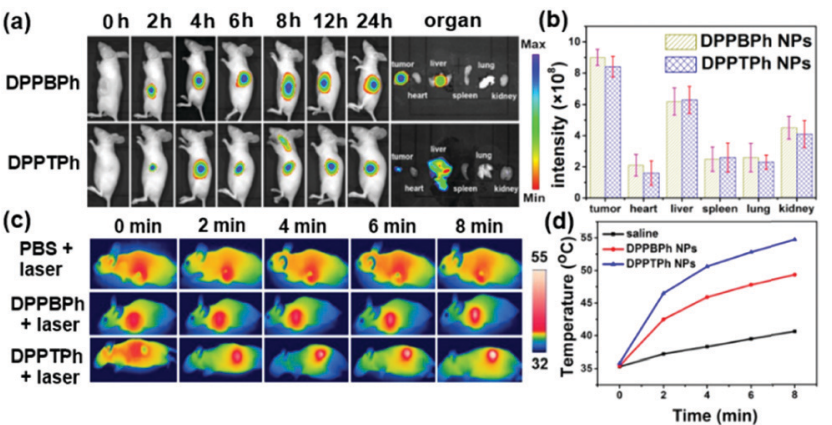

Fig. 5 (a) In vivo fluorescence imaging of nude mice after injection of DPPBPh and DPPTPh NPs at different times and ex vivo bio-distribution of the NPs in the tumor, heart, liver, spleen, lungs and kidneys. (b) Quantitation of the fluorescence intensity of the NPs in the tumor, heart, liver, spleen, lungs and kidneys after intravenous injection of DPPBPh and DPPTPh NPs for $24 \mathrm{~h}(n=3)$. (c) IR thermal images show the temperature change of tumor-bearing mice after injection with DPPBPh and DPPTPh NPs under irradiation, respectively, $\left(660 \mathrm{~nm}, 0.5 \mathrm{~W} \mathrm{~cm}^{-2}, 8 \mathrm{~min}\right)$. (d) Temperature increase of the tumor after intravenous injection of DPPBPh and DPPTPh NPs after 8 min irradiation $\left(660 \mathrm{~nm}, 0.5 \mathrm{~W} \mathrm{~cm}^{-2}\right)$.

the fluorescence intensity remained high for both of the two groups. The mice were sacrificed after treatment and the fluorescence intensity of the tumor, heart, liver, spleen, lungs and kidneys were quantitatively measured (Fig. 5b), showing the high tumor accumulation of the NPs via the EPR effect. To investigate the photothermal efficacy of these NPs, the temperature in the tumor site under laser irradiation was recorded by the IR camera. In the control group, the temperature increased from 35.2 to $38.6{ }^{\circ} \mathrm{C}$, while the temperatures of the DPPBPh and DPPTPh NPs groups were increased 12.3 and $19.5{ }^{\circ} \mathrm{C}$, respectively, suggesting the excellent photothermal effect (Fig. $5 \mathrm{c}$ and d). 


\section{Pharmacokinetics analysis and phototherapy}

A sensitive and specific ultraviolet spectrophotometer was applied in the pharmacokinetic study of DPPBPh, DPPTPh and the corresponding NPs after intravenous administration. The mean plasma concentration-time profiles of DPPBPh, DPPTPh and their NPs are shown in Fig. 6a. The major pharmacokinetic parameters were calculated by a non-compartmental model and are presented in Table $1 .^{31}$ The values of the $\mathrm{AUC}_{(0-t)} / \mathrm{AUC}_{(0-\infty)}$ were more than $80 \%$, suggesting that the blood collection time point was suitable for pharmacokinetic studies. The terminal elimination half-life $\left(T_{1 / 2}\right)$ of DPPBPh and DPPTPh were 2.126 and $3.317 \mathrm{~h}$, while that of the corresponding NPs were 6.851 and $7.362 \mathrm{~h}$, respectively. These data showed that the clearance of free drug was more quick than that of the NPs, and DPPTPh NPs could reside longer than DPPBPh (Fig. 6a, b and Table 1). The systematic clearance (CLz) of the free drug for DPPBPh and DPPTPh was
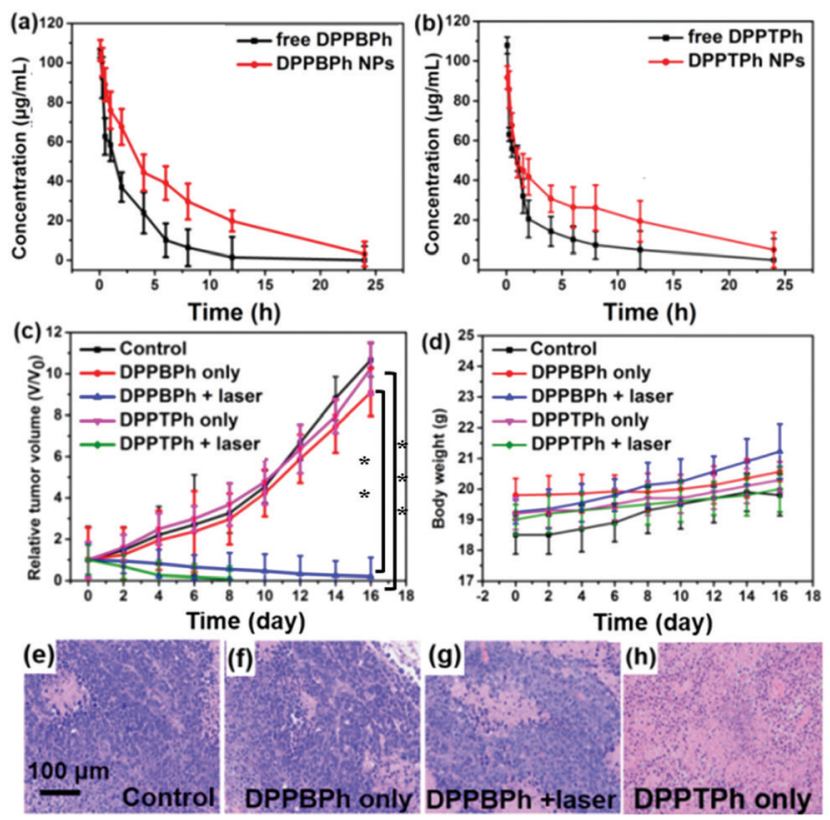

Fig. 6 ( $a$ and b) Time dependent drug concentration of DPPBPh and DPPTPh NPs in mice $(n=6)$. (c) Relative tumor volume ${ }^{* *} p<0.01$ and $* \star * p<0.001)$ and (d) body weight change during the treatment. $(e-h)$ H\&E stained pictures of the tumors of the control, DPPBPh only, DPPBPh + laser and DPPTPh only groups. Scale bar: $100 \mu \mathrm{m}$.

Table 1 Pharmacokinetics parameters of free or NPs of DPPBPh and DPPTPh

\begin{tabular}{lcccc}
\hline Parameter & Free DPPBPh & DPPBPh NPs & Free DPPTPh & DPPTPh NPs \\
\hline$T_{1 / 2}$ & 2.126 & 6.851 & 3.317 & 7.362 \\
$\operatorname{AUC}_{(0-t)}$ & 249.539 & 662.284 & 193.515 & 536.731 \\
$\operatorname{AUC}_{(0-\infty)}$ & 253.896 & 720.323 & 216.591 & 591.526 \\
$\operatorname{MRT}_{(0-t)}$ & 2.676 & 6.267 & 3.317 & 7.362 \\
$V_{\mathrm{Z}}$ & 0.121 & 0.137 & 0.14 & 0.092 \\
$\mathrm{CL}_{\mathrm{Z}}$ & 0.039 & 0.014 & 0.023 & 0.008
\end{tabular}

$T_{1 / 2}$ : terminal elimination half-life; $\mathrm{AUC}_{0-t}$ : the area under the curve from zero to the last measurable plasma concentration point; $\mathrm{AUC}_{0-\infty}$ : the area under the curve from zero to infinity; $\mathrm{MRT}_{(0-t)}$ : mean residence time; CLz: systemic clearance; $V_{\mathrm{Z}}$ : apparent volume of distribution.

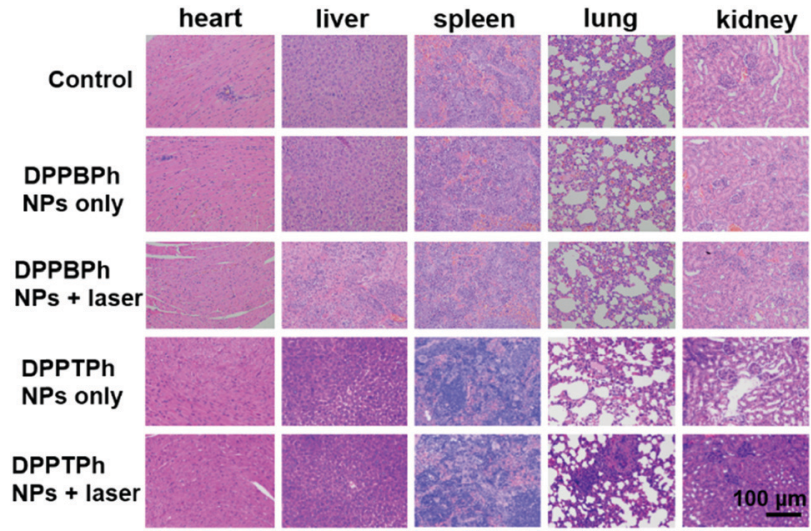

Fig. 7 H\&E stained images of the heart, liver, spleen, lungs, and kidneys after treatment.

0.039 and $0.023 \mathrm{~L} \mathrm{~h}^{-1} \mathrm{~kg}^{-1}$, respectively. While CLz of the DPPBPh and DPPTPh NPs was 0.014 and $0.008 \mathrm{~L} \mathrm{~h}^{-1} \mathrm{~kg}^{-1}$, respectively, indicating that the NPs were eliminated more slowly than the corresponding free drugs, and DPPBPh NPs eliminated quicker than DPPTPh NPs. All the results suggested that the NPS could extend the residence time in mice as compared with their corresponding free molecules.

As demonstrated in Fig. 6c, tumor growth was distinctly suppressed in the DPPBPh and DPPTPh NPs groups by laser irradiation. The relative tumor volume is 0.18 for the DPPBPh group with laser irradiation while for the DPPTPh, the tumor growth has been completely inhibited. These results demonstrated that DPPTPh NPs are superior to DPPBPh NPs in terms of phototherapy efficacy. For the no illumination groups, the relative tumor volume of DPPBPh NP treated mice (8.52) is lower than that of the DPPTPh NP group (10.23) and the control group (10.82), showing the higher dark toxicity of DPPBPh but negligible one of DPPTPh at high concentration, which is consistent with the MTT assay. No obvious body weight decay was observed, indicating the good biocompatibility of these NPs (Fig. 6d). After treatment, the mice were sacrificed for H\&E staining. The nuclei of the HeLa cells in the control group remain common. However, for the DPPBPh only group (Fig. 6e), a small proportion of the nuclei distorted and those of the irradiation group are severely destroyed (Fig. $6 f$ and g). For the DPPTPh only group, the nuclei resemble those of the control group, suggesting the low dark toxicity of DPPTPh (Fig. 6h). The tumors of the mice in the groups are shown in Fig. S7 (ESI $\dagger$ ). Furthermore, the H\&E stained photograph of the normal tissues, such as heart, liver, spleen, lungs and kidneys have also been illustrated in Fig. 7. The normal tissues suffer from no damage, showing that these NPs have no side effects on them and the good biosafety. ${ }^{32-41}$

\section{Conclusions}

In summary, we have synthesized two kinds of GSH responsive DPP derivatives, including DPPBPh and DPPTPh, and their corresponding NPs via the nanoprecipitation method for combination cancer phototherapy. The thiol group in GSH can react with 
the $-\mathrm{CN}$ group in DPPBPh and DPPTPh to form thiazole through the Michael addition reaction and recover the quenched fluorescence. In vivo fluorescence imaging reveals that the NPs can target and accumulate in the tumor sites by the EPR effect for GSH responsive turn-on fluorescence imaging. In particular, the DPPTPh NPs, with higher ${ }^{1} \mathrm{O}_{2}$ QY and photothermal conversion efficiency than DPPBPh NPs, exhibit remarkable efficiency in inhibiting tumor growth without adverse effects. The results provide a new approach to designing novel nanotheranostic agents for fluorescence "turn on" imaging guided PTT and PDT synergistic therapy.

\section{Conflicts of interest}

There are no conflicts to declare.

\section{Acknowledgements}

This work was supported by the NNSF of China (61525402, 61775095, 21704043 and 81872255), Jiangsu Provincial key research and development plan (BE2017741), Six talent peak innovation team in Jiangsu Province (TD-SWYY-009 and WSW041), the NSF of Jiangsu Province (BK20170990 and 17KJB150020), and Key medical talents fund of Jiangsu Province (2016KJQWZDRC-03).

\section{Notes and references}

1 W. P. Fan, P. Huang and X. Y. Chen, Chem. Soc. Rev., 2016, 45, 6488-6519.

2 Y. J. Liu, P. Bhattarai, Z. F. Dai and X. Y. Chen, Chem. Soc. Rev., 2019, 48, 2053-2108.

3 J. H. Zou, Z. H. Yin, K. K. Ding, Q. Y. Tang, J. W. Li, W. L. Si, J. J. Shao, Q. Zhang, W. Huang and X. C. Dong, ACS Appl. Mater. Interfaces, 2017, 9, 32475-32481.

4 J. H. Zou, Z. H. Yin, P. Wang, D. P. Chen, J. J. Shao, Q. Zhang, L. G. Sun, W. Huang and X. C. Dong, Chem. Sci., 2018, 9, 2188-2194.

5 S. Y. Ye, J. M. Rao, S. H. Qiu, J. L. Zhao, H. He, Z. L. Yan, T. Yang, Y. B. Deng, H. T. Ke, H. Yang, Y. L. Zhao, Z. Q. Guo and H. B. Chen, Adv. Mater., 2018, 30, 1801216.

6 Y. Y. Yuan, C. J. Zhang, M. Gao, R. Y. Zhang, B. Z. Tang and B. Liu, Angew. Chem., Int. Ed., 2015, 54, 1780-1786.

7 J. H. Zou, P. Wang, Y. Wang, G. Y. Liu, Y. W. Zhang, Q. Zhang, J. J. Shao, W. L. Si, W. Huang and X. C. Dong, Chem. Sci., 2019, 10, 268-276.

8 M. Li, Y. Gao, Y. Y. Yuan, Y. Z. Wu, Z. F. Song, B. Z. Tang, B. Liu and Q. C. Zheng, ACS Nano, 2017, 11, 3922-3932.

9 Z. Yang, W. P. Fan, W. Tang, Z. Y. Shen, Y. L. Dai, J. B. Song, Z. T. Wang, Y. Liu, L. S. Lin, L. L. Shan, Y. J. Liu, O. Jacobson, P. F. Rong, W. Wang and X. Y. Chen, Angew. Chem., Int. Ed., 2018, 57, 14101-14105.

10 C. R. Gordijo, A. Z. Abbasi, M. Ali Amini, H. Y. Lip, A. Maeda, P. Cai, P. J. O'Brien, R. S. DaCosta, A. M. Rauth and X. Y. Wu, Adv. Funct. Mater., 2015, 25, 1858-1872.

11 Y. Cai, Q. Y. Tang, X. J. Wu, W. L. Si, Q. Zhang, W. Huang and X. C. Dong, ACS Appl. Mater. Interfaces, 2016, 8, 10737-10742.
12 Y. Cai, Q. Y. Tang, X. J. Wu, W. L. Si, W. Huang, Q. Zhang and X. C. Dong, ChemistrySelect, 2016, 12, 3071-3074.

13 Z. W. Wei, M. Wu, S. Y. Lan, J. Li, X. L. Zhang, D. Zhang, X. L. Liu and J. F. Liu, Chem. Commun., 2018, 54, 13599-13602.

14 J. Wang, Y. D. Hang, H. Q. Tan, T. Jiang, X. Qu and J. L. Hua, J. Photochem. Photobiol., A, 2017, 346, 265-272.

15 Y. Cai, P. P. Liang, Q. Y. Tang, X. Y. Yang, W. L. Si, W. Huang, Q. Zhang and X. C. Dong, ACS Nano, 2017, 11, 1054-1106.

16 X. Chen, Y. Zhou, X. Peng and J. Yoon, Chem. Soc. Rev., 2010, 39, 2120-2135.

17 B. Tang, L. Yin, X. Wang, Z. Chen, L. Tong and K. Xu, Chem. Commun., 2009, 5293-5295.

18 H. Guo, Y. Jing, X. Yuan, S. Ji, J. Zhao, X. Li and Y. Kan, Org. Biomol. Chem., 2011, 9, 3844-3853.

19 J. Shao, H. Guo, S. Ji and J. Zhao, Biosens. Bioelectron., 2011, 26, 3012-3017.

20 S. Ji, J. Yang, Q. Yang, S. Liu, M. Chen and J. Zhao, J. Org. Chem., 2009, 74, 4855-4865.

21 S. Ji, H. Guo, X. Yuan, X. Li, H. Ding, P. Gao, C. Zhao, W. Wu, W. Wu. and J. Zhao, Org. Lett., 2010, 12, 2876-2879.

22 W. Lin, L. Long, L. Yuan, Z. Cao, B. Chen and W. Tan, Org. Lett., 2008, 10, 5577-5580.

23 J. Bouffard, Y. Kim, T. M. Swager, R. Weissleder and S. A. Hilderbrand, Org. Lett., 2008, 10, 37-40.

24 R. Zhang, X. Yu, Z. Ye, G. Wang, W. Zhang and J. Yuan, Inorg. Chem., 2010, 49, 7898-7903.

25 L. Xiong, Q. Zhao, H. Chen, Y. Wu, Z. Dong, Z. Zhou and F. Li, Inorg. Chem., 2010, 49, 6402-6408.

26 X. Chen, S. Ko, M. J. Kim, I. Shin and J. Yoon, Chem. Commun., 2010, 46, 2751-2753.

27 H. Li, J. Fan, J. Wang, M. Tian, J. Du, S. Sun, P. Sun and X. Peng, Chem. Commun., 2009, 5904-5906.

28 X. Li, S. Qian, Q. He, B. Yang, J. Li and Y. Hu, Org. Biomol. Chem., 2010, 8, 3627-3630.

29 T. Matsumoto, Y. Urano, T. Shoda, H. Kojima and T. Nagano, Org. Lett., 2007, 9, 3375-3377.

30 L. Deng, W. T. Wu, H. M. Guo, J. Z. Zhao, S. M. Ji, X. Zhang, X. L. Yuan and C. L. Zhang, J. Org. Chem., 2011, 76, 9294-9304.

31 J. Zhu, B. Xue, B. Ma, Q. Zhang, M. Liu, L. Liu, D. Yao, H. Qi, Y. Wang, H. Ying and Z. Wu, J. Chromatogr. B: Anal. Technol. Biomed. Life Sci., 2015, 993, 47-59.

32 Z. Yang, R. Tian, J. Wu, Q. L. Fan, B. C. Yung, G. Niu, O. Jacobson, Z. T. Wang, G. Liu, G. Yu, W. Huang, J. B. Song and X. Y. Chen, ACS Nano, 2017, 11, 4247-4255.

33 B. B. Gu, W. B. Wu, G. X. Xu, G. X. Feng, F. Yin, P. H. Joo Chong, J. L. Qu, K. T. Yong and B. Liu, Adv. Mater., 2017, 1701076.

34 J. Shen, J. J. Chen, Z. Ke, D. F. Zou, L. G. Sun and J. H. Zou, Mater. Chem. Front., 2019, 3, 1123-1127.

35 L. Huang, Z. J. Li, Y. Zhao, J. Y. Yang, Y. C. Yang, A. I. Pendharkar, Y. W. Zhang, S. Kelmar, L. Y. Chen, W. T. Wu, J. Z. Zhao and G. Han, Adv. Mater., 2017, 29, 1604789.

36 C. J. Zhang, G. X. Feng, S. D. Xu, Z. S. Zhu, X. M. Lu, J. Wu and B. Liu, Angew. Chem., Int. Ed., 2016, 55, 6192-6196.

37 W. Sun, S. Y. Li, B. Häupler, J. Liu, S. B. Jin, W. Steffen, U. S. Schubert, H. J. Butt, X. J. Liang and S. Wu, Adv. Mater., 2017, 29, 1603702 . 
38 Z. Yang, Y. L. Dai, C. Yin, Q. L. Fan, W. Zhang, J. B. Song, G. C. Yu, W. Tang, W. P. Fan, B. C. Yung, J. Li, X. Li, X. Li, Y. Tang, W. Huang, J. B. Song and X. Y. Chen, Adv. Mater., 2018, 30, 1707509.

39 J. Yang, X. Q. Gu, W. T. Su, X. Y. Hao, Y. J. Shi, L. Y. Zhao, D. F. Zou, G. W. Yang, Q. Y. Li and J. H. Zou, Mater. Chem. Front., 2018, 2, 1842-1846.
40 Z. Yang, J. B. Song, W. Tang, W. P. Fan, Y. L. Dai, Z. Y. Shen, L. S. Lin, S. Cheng, Y. Liu, G. Niu, P. Rong, W. Wang and X. Y. Chen, Theranostics, 2019, 9, 526.

41 Z. Yang, Y. L. Dai, L. Shan, Z. Y. Shen, Z. T. Wang, B. C. Yung, O. Jacobson, Y. Liu, W. Tang, S. Wang, L. S. Lin, G. Niu, P. Huang and X. Y. Chen, Nanoscale Horiz., 2019, 4, 426-433. 